\title{
Recurrent Cerebral Astroblastoma with Aggressive Histology: An Uncommon Case
}

\author{
${ }^{1}$ Department of Neurosurgery, All India Institute of Medical \\ Sciences, Bhubaneswar, Odisha, India \\ ${ }^{2}$ Department of Pathology and Laboratory Medicine, All India \\ Institute of Medical Sciences, Bhubaneswar, Odisha, India \\ Indian J Neurosurg 2018;7:150-154
}

Sumit Bansal ${ }^{1}$ Susama Patra² Pritinanda Mishra² Ashok Kumar Mahapatra ${ }^{1}$

\begin{abstract}
Address for correspondence Dr. Sumit Bansal, MCh, Room No. 419, Department of Neurosurgery, All India Institute of Medical Sciences, Bhubaneswar, Odisha 751019, India (e-mail: drsumitbansal@gmail.com).
\end{abstract}

\begin{abstract}
Keywords

- astroblastoma

- malignant

- cystic

Astroblastomas are uncommon tumors of uncertain histogenesis. Recently tanycyte is postulated to be the cell of origin. These occur predominantly in the cerebral hemisphere of young adults and children. Astroblastoma constitutes approximately 0.45 to $2.8 \%$ of all neuroglial tumors. We are reporting a case of 28 -year-old woman who presented with headache and double vision. Magnetic resonance imaging (MRI) of the brain revealed well-demarcated, ring-enhancing solid cystic mass in right temporoparietal lobe with significant mass effect. The patient underwent gross total resection of the lesion through right temporoparietal craniotomy. The histopathologic diagnosis was suggestive of astroblastoma. She was advised for postoperative radiotherapy, but she lost to follow-up and again presented 1 year later with recurrent tumor in the same location along with another lesion in infratentorial location. She was reoperated and gross total excision of supra- and infratentorial tumor was done. She also received radiotherapy. We are reporting this uncommon case of astroblastoma and also reviewing the existing literature.
\end{abstract}

\section{Introduction}

Astroblastomas are unusual tumors, constituting 0.45 to $2.8 \%$ of all neuroglial tumors. ${ }^{1}$ The cell of origin of astroblastoma is controversial. It was considered to arise from the embryonic cell programmed to become astrocyte. Presence of intermediate filament on ultrastructure shows ependymal differentiation, along with positive staining for glial fibrillary acidic protein (GFAP) and S100 suggesting the possibility of a cell with features between astrocytes an ependymal cells. Because of the rarity and lack of sufficient clinical-pathologic data, it has not been included in the World Health Organization (WHO) grading system. They occur predominantly in the cerebral hemisphere of young adults and children. ${ }^{2}$ Existing literature is limited with few case reports. ${ }^{3.4}$ We report a rare case of astroblastoma with recurrence after 1 year.

\section{Case Report}

A 28-year-old woman presented to us with holocranial headache and double vision for 2 months. Her neurologic examination was normal. Magnetic resonance imaging (MRI) of the brain revealed well-demarcated, ring-enhancing solid cystic mass in right temporoparietal lobe abutting superior surface of tentorium with mass effect. The patient underwent gross total resection of the lesion through right temporoparietal craniotomy ( - Fig. 1). Histopathology showed a highly cellular papillary neoplasm composed of polygonal cells with abundant eosinophilic cytoplasm and round to oval pleomorphic nuclei. Admixed are several large multinucleate cells. The mitotic index was 5/10 HPF (high-power field). There were areas of cystic change, hemorrhage, and infarct-like necrosis. On immunohistochemistry, tumor cells were variably positive received

May 4, 2016

accepted

January 23, 2017

published online

April 18, 2017
DOI https://doi.org/

10.1055/s-0037-1601358.

ISSN 2277-954X.
(C2018 Neurological Surgeons' Society of India
License terms

()(1) $\Theta \circledast$ 


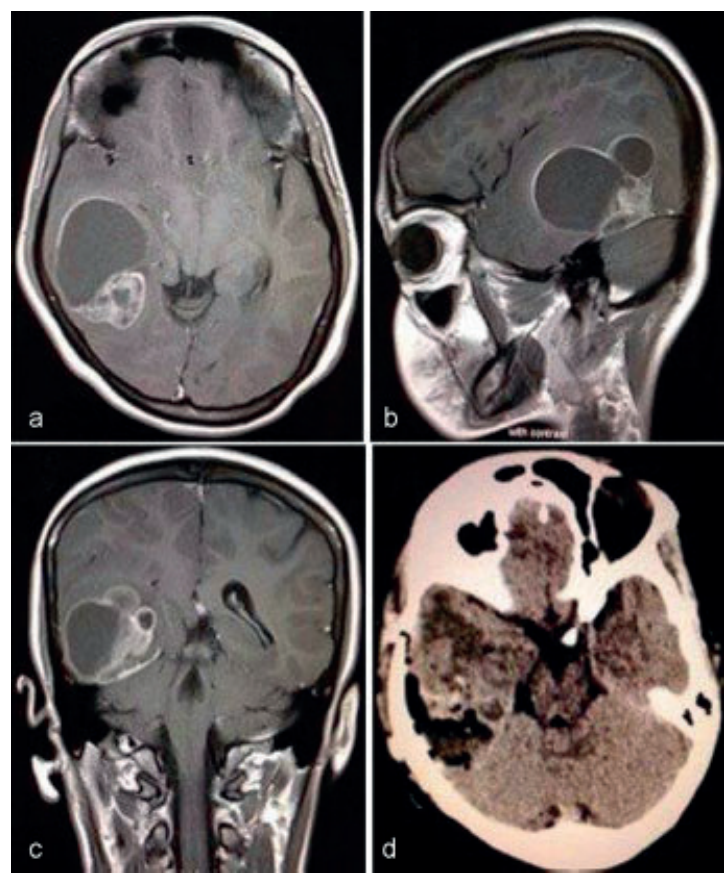

Fig. 1 First surgery: Contrast MRI of the brain showing solid cystic lesion in right temporoparietal region abutting supratentorial surface in axial (a), sagittal (b), and coronal (c) views. (d) Postoperative CT scan (axial view) showing gross total excision of tumor. for GFAP; diffusely positive for S100 and vimentin; and negative for synaptophysin, progesterone receptor (PR), and P53. Epithelial membrane antigen (EMA) shows membrane positivity in focal areas, staining group of cells. There was no dot positivity. MIB-1 labeling index was approximately $15 \%$ in the cellular zones. The histopathologic diagnosis of high-grade as-

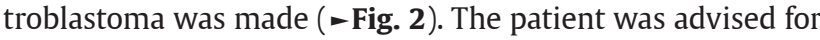
postoperative radiotherapy, but she lost to follow-up.

After 1 year, the patient presented to us with headache only. MRI of the brain showed recurrent tumor in the same location with infratentorial extension. She was reoperated, and gross total excision of supra- and infratentorial tumor was done ( $\boldsymbol{- \text { Fig. }} \mathbf{3}$ ). The recurrent tumor showed similar morphology but with increased pleomorphism, multinucleation, and brisk mitotic figures of both typical and atypical forms with an average of 15/10 HPF. There were large areas of hemorrhage and infarct-like necrosis. Focal areas of calcification mostly involving small-caliber vessels and stromal sclerosis are also noted. Immunohistochemistry revealed marked reduction in GFAP expression of tumor cells compared with the initial tumor. Staining pattern of the remaining markers (S100, vimentin, synaptophysin, progesterone receptor, and EMA) was same as before. Cells were negative for pancytokeratin and desmin. MIB-1 labeling index was approximately $25 \%$ in cellular zones. More than $50 \%$ of the cells showed

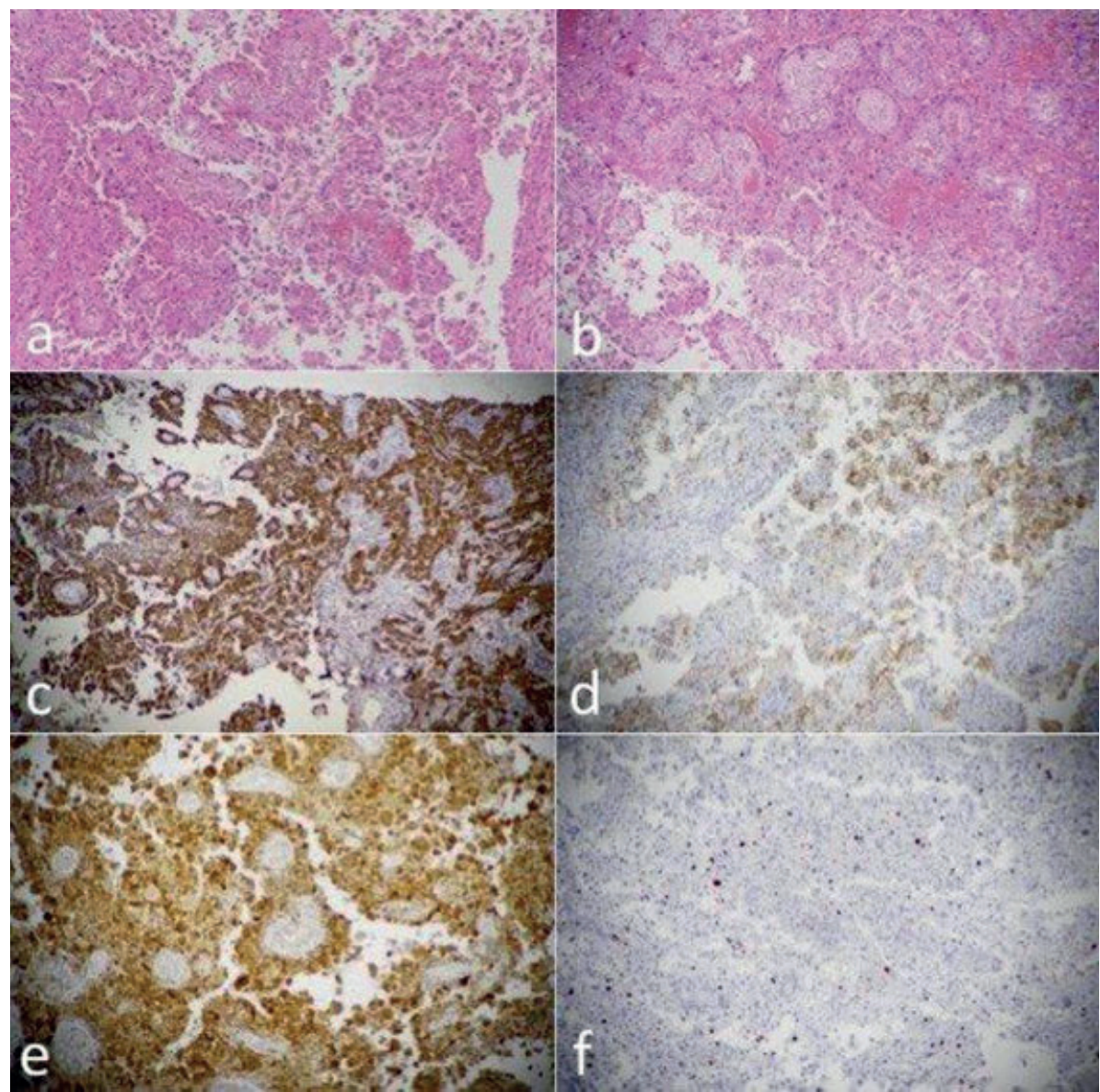

Fig. 2 (a) Papillary tumor (10x H\&E). (b) Fibrovascular core with hyalinization and perivascular arrangement of tumor cells (20x H\&E). Immunohistochemistry (IHC). (c) Diffuse expression of GFAP by tumor cells. (d) Focal positivity by EMA. (e) Diffuse positivity by S-100. (f) MIB-1 index is $15 \%$ in cellular areas. 


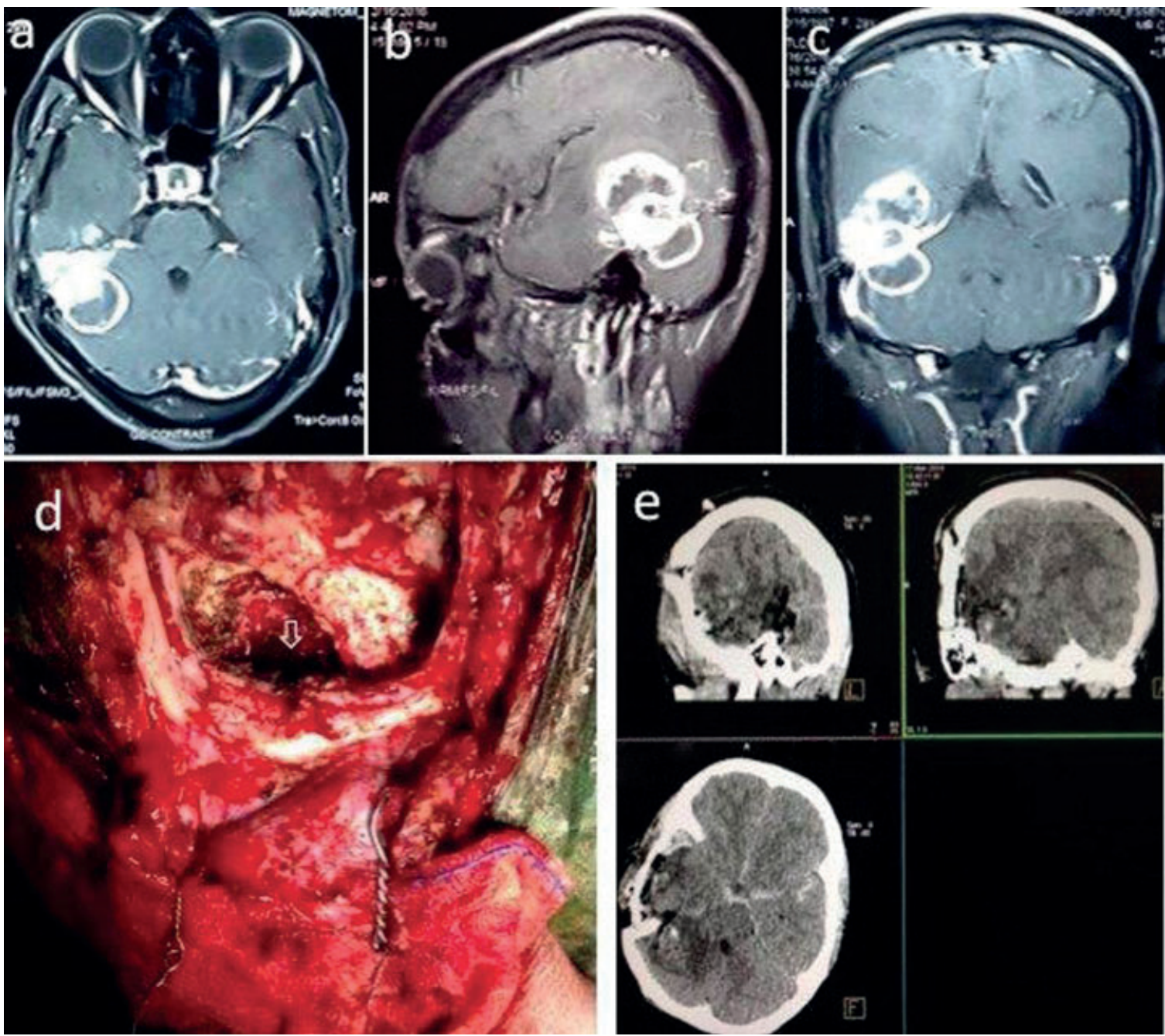

Fig. 3 Second surgery: Contrast MRI of the brain showing recurrent tumor in right temporoparietal region with infratentorial extension in axial (a), sagittal (b), and coronal (c) views. (d) Intraoperative image showing gross tumor excision in both supra- and infratentorial locations (arrow). (e) Postoperative CT of the head showing gross total excision of tumor from both supra- and infratentorial locations.

nuclear positivity for P53. The final diagnosis was that of high-grade astroblastoma with malignant transformation based on the high proliferative index and expression of P53 in more than $50 \%$ of cells ( - Fig. 4). Postoperatively, she received radiotherapy and on regular follow-up.

\section{Discussion}

Bailey and Cushing ${ }^{5}$ first described these lesions in 1926, and Bailey and Bucy ${ }^{6}$ supported their statistical analysis in 1930. They believed that astroblastoma originated from astroblasts, an intermediate stage between glioblasts and astrocytes. These are rare glial tumors usually located in the cerebral hemisphere. However, tumor invasion has also been reported into the corpus callosum, cerebellum, brainstem, and optic nerve. ${ }^{7}$ The studies performed till date show striking female preponderance with a male-to-female ratio of $1: 11 .^{8}$ Most commonly patients present with features of raised intracranial tension, drowsiness, and seizures. Astroblastomas are mostly reported in children and young adults, but congenital cases have also been reported rarely. ${ }^{1}$
Bell et $\mathrm{al}^{8}$ in a series of 12 cases of astroblastomas reported that astroblastomas are almost exclusively reported supratentorially and they are peripheral in location with both solid and cystic components. Largest series till date by Ahmad et $\mathrm{al}^{9}$ described supratentorial tumor location, older age, and treatment prior to 1990 as poor prognostic factors. Another series by Asha et $\mathrm{al}^{10}$ reported single-institute series of nine cases with recurrence in one case after 12 months after first surgery as in our case. Our case showed typical solid cystic lesion with ring enhancement similarly. Based on imaging, the differential diagnoses for astroblastomas include high-grade astrocytoma, pilocytic astrocytoma, primitive neuroectodermal tumor, ependymoma, and atypical teratoid-rhabdoid tumor. Unlike in high-grade tumors, perilesional edema is usually less in astroblastomas, including high-grade variant.

Astroblastomas are defined histologically by the presence of perivascular pseudorosettes and prominent perivascular hyalinization. ${ }^{11}$ They may resemble astrocytic tumors, ependymomas, and non-neuroepithelial tumors due to their astroblastic components. Lack of fibrillarity is an essential feature in distinguishing astroblastomas from other glial 

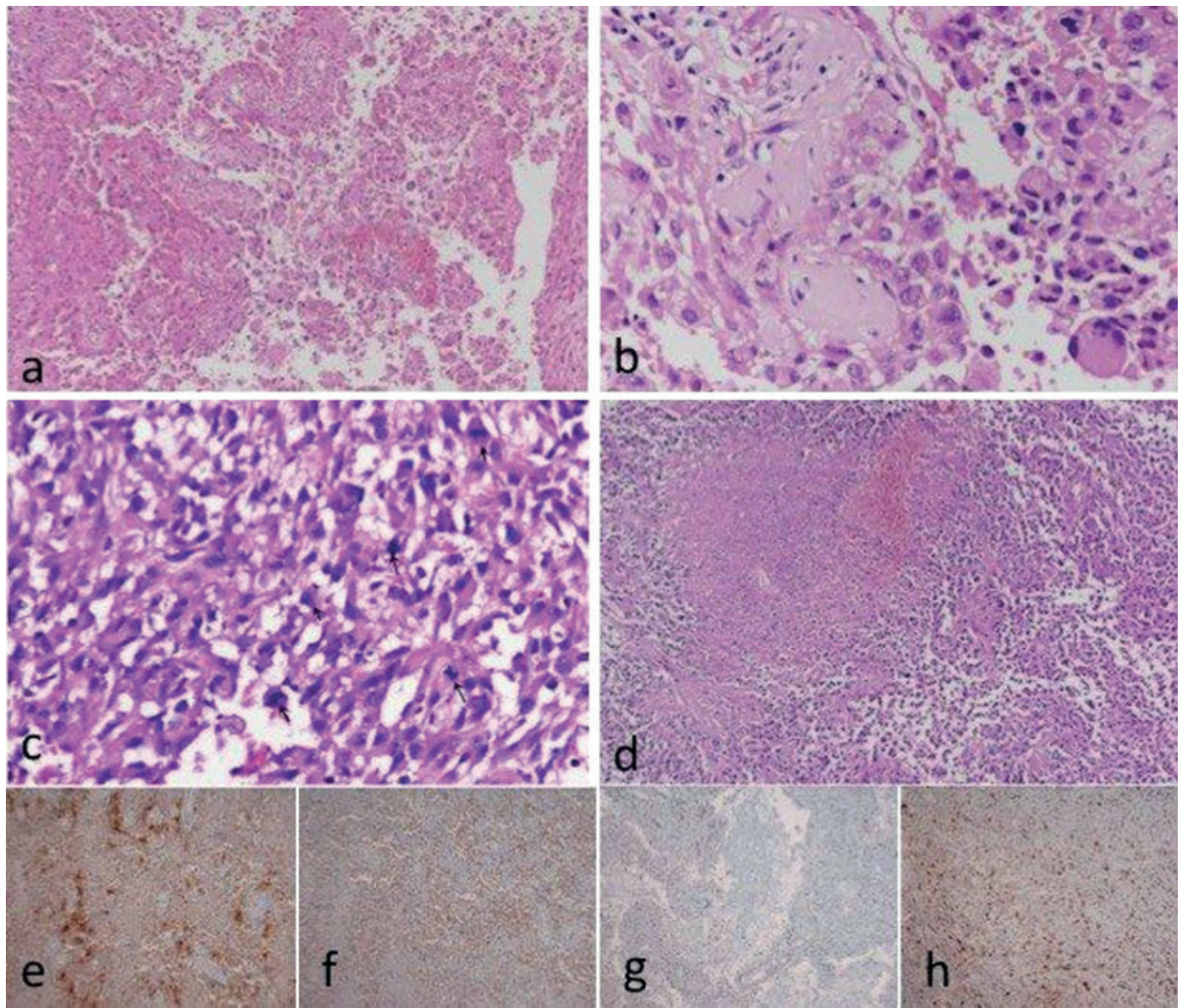

Fig. 4 (a) Recurrent tumor with similar morphology and (b) sclerosis of the papillary core (10x H\&E). (c) Higher magnification showing mitosis (20x H\&E). (d) Patchy areas of necrosis (10x H\&E). Immunohistochemistry (IHC). (e) Decreased expression of GFAP by the tumor cells. (f) EMA. (g) Expression of P53 by the tumor cells. (h) MIB-1 labeling index is $30 \%$ in cellular areas.

neoplasms. Immunohistochemically, astroblastomas are immunoreactive for GFAP, S100 protein, vimentin, and majority display a focal cytoplasmic immunoreactivity for EMA. Similar immunophenotypes were noted in the initial as well as recurrent tumor in the case of concern.

Astroblastomas along with gliomatosis cerebri and polar spongioblastoma are included in neuroepithelial tumors of uncertain origin and are grade 4 tumors as per 2007 contrary to grade 1 in WHO classification of brain tumors. Bonnin and Rubinstein ${ }^{7}$ reported two distinct histologic types: A lowgrade type with better differentiated pattern and favorable postoperative prognosis and a high-grade type showing more anaplastic microscopic features with short postoperative survival. High-grade lesions show focal or multifocal regions of high cellularity, anaplastic nuclear features, elevated mitotic indices, vascular proliferation, and necrosis with pseudopalisading. Our case was considered in high-grade group. However, pseudopalisading and microvascular proliferation were not present.
The present case of concern had proliferative indices exceeding the cutoff values for high-grade tumor as per WHO 2016 in both primary and in recurrent tumors. Apart from the high proliferative index, recurrent tumor showed additional features patchy areas of necrosis and expression of p53. However, the effect of the high proliferative index on outcome is still not clear.

Natural history of astroblastoma seems to place it in between astrocytoma and glioblastoma. ${ }^{12}$ Total resection is the best way of treating an astroblastoma. ${ }^{13}$ Favorable prognosis is almost always associated with well-circumscribed tumors, which permits total resection..$^{14}$ Given the peripheral location of astroblastomas, total resection seems a goal easier to achieve than in other gliomas. Regular follow-up is required even in low-grade variants due to unpredictable behavior. Adjuvant radiotherapy is recommended for high-grade and recurrent cases. ${ }^{15}$ Caroli et al ${ }^{14}$ reported a high-grade astroblastoma with a 5-year survival without recurrence after total resection, radiation therapy, and temozolomide usage. 
The lack of enough information about the natural history favors the use of adjuvant radiotherapy from the beginning.

\section{Conclusion}

Astroblastomas are very rare. Complete excision without radiotherapy and close follow-up is required in low-grade variants. High-grade and recurrent tumors should receive adjuvant radiotherapy. As per WHO 2016, gross total resection of even high-grade astroblastoma may result in favorable outcome.

\section{Financial Support}

None.

\section{Conflict of Interest}

None.

\section{References}

1 Pizer BL, Moss T, Oakhill A, Webb D, Coakham HB. Congenital astroblastoma: an immunohistochemical study. Case report. J Neurosurg 1995;83(03):550-555

2 Port JD, Brat DJ, Burger PC, Pomper MG. Astroblastoma: radiologic-pathologic correlation and distinction from ependymoma. AJNR Am J Neuroradiol 2002;23(02):243-247

3 Singh DK, Singh N, Singh R, Husain N. Cerebral astroblastoma: a radiopathological diagnosis. J Pediatr Neurosci 2014;9(01):45-47

4 Agarwal V, Mally R, Palande DA, Velho V. Cerebral astroblastoma: A case report and review of literature. Asian J Neurosurg 2012;7(02):98-100
5 Bailey P, Cushing H. A Classification of the Tumors of the Glioma Group on a Histogenetic Basis with a Correlated Study of Prognosis. Philadelphia, PA: J.B. Lippincott Company: 1926:134-167

6 Bailey P, Bucy PC. Astroblastomas of the brain. Acta Psychiatr Neurol 1930;5:439-461

7 Bonnin JM, Rubinstein LJ. Astroblastomas: a pathological study of 23 tumors, with a postoperative follow-up in 13 patients. Neurosurgery 1989;25(01):6-13

8 Bell JW, Osborn AG, Salzman KL, Blaser SI, Jones BV, Chin SS. Neuroradiologic characteristics of astroblastoma. Neuroradiology 2007;49(03):203-209

9 Ahmed KA, Allen PK, Mahajan A, Brown PD, Ghia AJ. Astroblastomas: a Surveillance, Epidemiology, and End Results (SEER)based patterns of care analysis. World Neurosurg 2014; 82(1-2): e291-e297

10 Asha U, Mahadevan A, Sathiyabama D, et al. Lack of IDH1 mutation in astroblastomas suggests putative origin from ependymoglial cells? Neuropathology 2015;35(04):303-311

11 McLendon RE, Enterline DS, Tien RD, Thorstad WL, Bruner JM. Astroblastomas. Bigner DD, McLendon RE, Bruner JM. Russell and Rubinstein's Pathology of Tumors of Nervous System. 6th ed. London, UK: Arnold; 1998:419-426

12 Burger PC, Scheithauer BW. Atlas of Tumor Pathology. Tumor of the Central Nervous System. Washington, DCArmed Forces Institute of Pathology; 1994:146-148

13 Cabello A, Madero S, Castresana A, Diaz-Lobato R. Astroblastoma: electron microscopy and immunohistochemical findings: case report. Surg Neurol 1991;35(02):116-121

14 Caroli E, Salvati M, Esposito V, Orlando ER, Giangaspero F. Cerebral astroblastoma. Acta Neurochir (Wien) 2004; 146(06):629-633

15 Unal E, Koksal Y, Vajtai I, Toy H, Kocaogullar Y, Paksoy Y. Astroblastoma in a child. Childs Nerv Syst 2008;24(02):165-168 